Brief Report

\title{
Mesh-Type Three-Dimensional (3D) Printing of Human Organs and Tumors: Fast, Cost-Effective, and Personalized Anatomic Modeling of Patient-Oriented Visual Aids
}

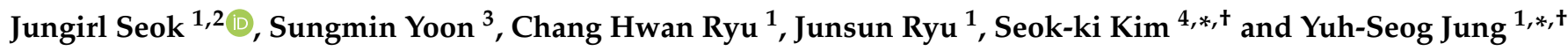 \\ 1 Department of Otorhinolaryngology-Head and Neck Surgery, National Cancer Center, Goyang 10408, Korea; \\ junn279@gmail.com (J.S.); changhwanr@ncc.re.kr (C.H.R.); jsryu@ncc.re.kr (J.R.) \\ 2 Department of Biomedical Engineering, College of Medicine, Seoul National University, Seoul 03080, Korea \\ 3 Division of Convergence Technology, National Cancer Center, Goyang 10408, Korea; \\ sungmin1551@naver.com \\ 4 Department of Nuclear Medicine, National Cancer Center, Goyang 10408, Korea \\ * Correspondence: skkim@ncc.re.kr (S.-k.K.); jysorl@ncc.re.kr (Y.-S.J.); Tel.: +82-31-920-1731 (S.-k.K.); \\ +82-31-920-1685 (Y.-S.J.); Fax: +82-31-920-2799 (S.-k.K. \& Y.-S.J.) \\ + Authors contributing equally to this work.
}

check for updates

Citation: Seok, J.; Yoon, S.; Ryu, C.H.; Ryu, J.; Kim, S.-k.; Jung, Y.-S.

Mesh-Type Three-Dimensional (3D) Printing of Human Organs and Tumors: Fast, Cost-Effective, and Personalized Anatomic Modeling of Patient-Oriented Visual Aids. Appl. Sci. 2021, 11, 1047. https://doi.org/ 10.3390/app11031047

Received: 19 December 2020

Accepted: 21 January 2021

Published: 25 January 2021

Publisher's Note: MDPI stays neutra with regard to jurisdictional claims in published maps and institutional affiliations.

Copyright: (c) 2021 by the authors. Licensee MDPI, Basel, Switzerland. This article is an open access article distributed under the terms and conditions of the Creative Commons Attribution (CC BY) license (https:/ / creativecommons.org/licenses/by/ $4.0 /)$.
Abstract: Although three-dimensional (3D)-printed anatomic models are not new to medicine, the high costs and lengthy production times entailed have limited their application. Our goal was developing a new and less costly 3D modeling method to depict organ-tumor relations at faster printing speeds. We have devised a method of 3D modeling using tomographic images. Coordinates are extracted at a specified interval, connecting them to create mesh-work replicas. Adjacent constructs are depicted by density variations, showing anatomic targets (i.e., tumors) in contrasting colors. An array of organ solid-tumor models was printed via a Fused Deposition Modeling 3D printer at significantly less cost $\left(\$ 0.05 / \mathrm{cm}^{3}\right)$ and time expenditure $\left(1.73 \mathrm{~min} / \mathrm{cm}^{3}\right.$; both, $p<0.001)$. Printed models helped promote visual appreciation of organ-tumor anatomy and adjacent tissues. Our mesh-work 3D thyroidal prototype reproduced glandular size/contour and tumor location, readily approximating the surgical specimen. This newly devised mesh-type 3D printing method may facilitate anatomic modeling for personalized care and improve patient awareness during informed surgical consent.

Keywords: three-dimensional (3D) printing; anatomic modeling; personalized medicine

\section{Introduction}

To obtain patient consent for an operation, the nature of the procedure must be explained, as well as expected benefits and potential risks or adverse effects, alternative treatment options, and likely outcomes if surgery is declined [1]. Appropriate and welldocumented informed consent is mandatory to preclude civil action for negligence or otherwise protect medical staff from legal conflicts [2,3]. Unfortunately, attempts to convey the fundamentals of intended procedures sometimes fail, depriving patients of proper working knowledge for granting consent [4]. There are reports that visual aids used to supplement verbal descriptions allow better conceptualization and processing of the information delivered [5,6]. Three-dimensional (3D) models, in particular, may depict the visual aspects of procedures more effectively. Recently, 3D printers have been studied expressly for this purpose [7-9].

3D-printed anatomic models are widely used in surgical fields, particularly in orthopedic and maxillofacial specialties, as operative guides or templates for surgical planning, implant design, molding of prosthetics, and patient selection [10]. They have also been assets in patient education [7,8]. Personalized 3D models that replicate actual organ or tissue conditions may aid in evaluating disease states and help expedite preoperative 
preparations [11]. However, such models have not yet proven cost-effective, the expense and time invested acknowledged as shortcomings $[7,8,10]$.

The present study was undertaken to preliminarily investigate a mesh-type 3D modeling technique for solid tumors that effectively reduces cost and printing time, without loss of organ-tumor anatomic relations.

\section{Methods}

\subsection{Ethical Considerations}

The institutional review board at the National Cancer Center, Korea, approved our study protocol (IRB No. NCC2020-0248). No patient data was collected, so required informed consent was waived.

\subsection{Personalized Mesh-Type 3D Modeling and Two-Color 3D Printing}

Our newly devised modeling method was intended to limit unnecessary input while ensuring the accuracy of organ-tumor anatomic depictions. Normal structures are portrayed in one color (i.e., white) as differing mesh densities, with tumors shown in sharply contrasting hues (i.e., blue) (Figure 1).
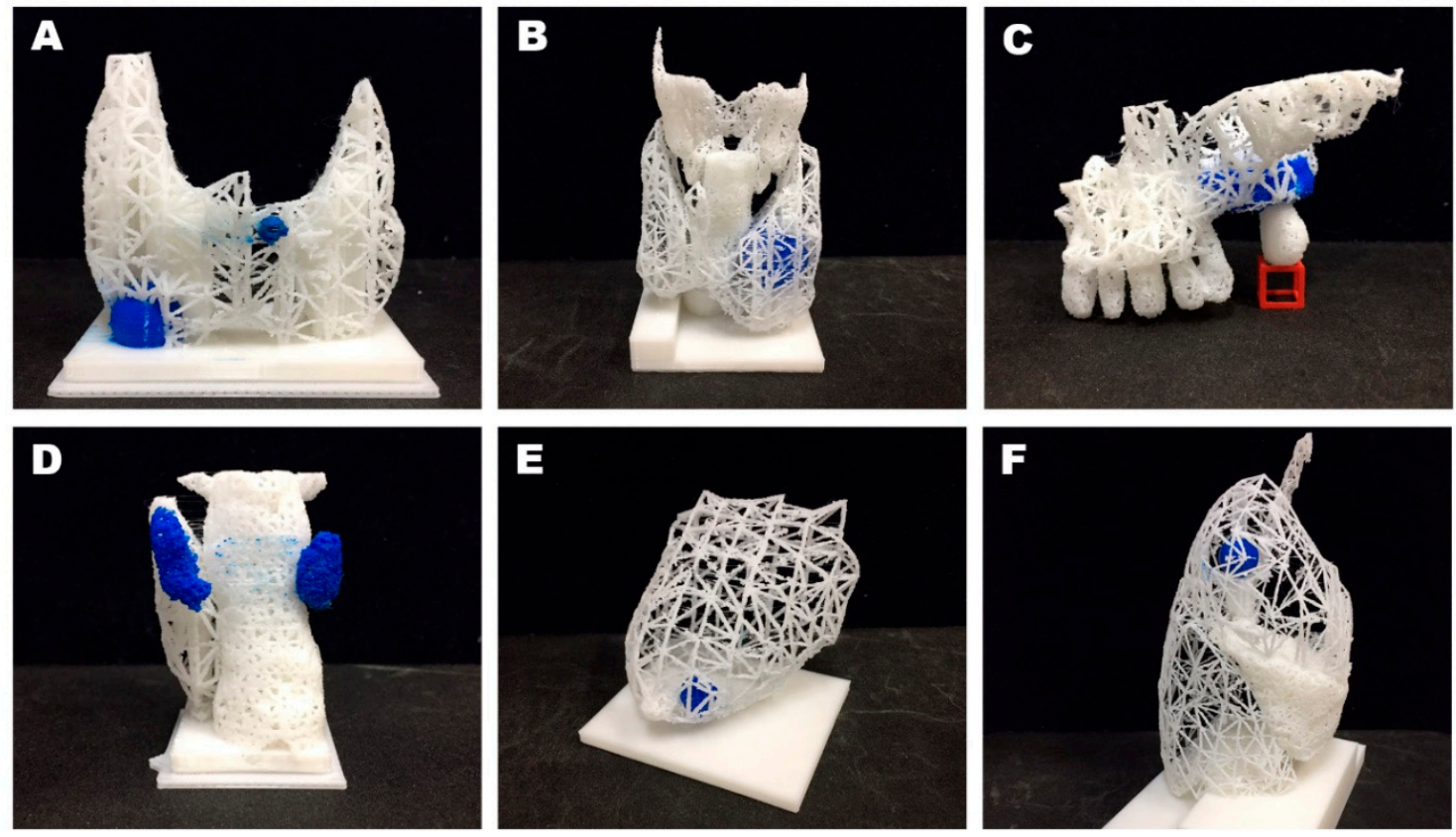

Figure 1. Examples of mesh-type three-dimensional (3D) modeling: (A) Tumors of thyroid gland; (B) thyroid gland and adjacent cartilage; (C) maxillary tumor (lateral view); (D) parathyroid adenoma (posterior view); (E) breast tumor; and (F) right lung nodule (lateral view). Note: each tumor printed in blue, adjacent structures shown as differing mesh densities.

The segmentation of organs and tumors in Digital Imaging and Communications in Medicine (DICOM) images may be done manually or using deep learning techniques [12]. To confirm the feasibility of this mesh-type 3D modeling, we prepared images through manual segmentation. A segmented area or 'mask' was expressed as a two-dimensional array in programming language. Masks were then stacked to build 3D arrays and resampled to reflect actual sizes, based on original pixel spacing and slice thickness. The final step was the marking of tumor locations and areas. All 3D arrays were subsequently reconfigured to 3D representations in triangular meshes, using the Trimesh package (v2.38.24) in Python language [13]. After smoothing, the density of every structure was set by stipulating one vertex per designated area (e.g., $4.5 \mathrm{~mm}^{2}$ ). Using the PyQt5 library (v5.15.0, Riverbank Computing Ltd., Dorchester, UK), an application capable of handling the entire process 
was invoked to do so (Figure 2). Such vertex/facet restrictions enabled completion of our mesh-type 3D models.
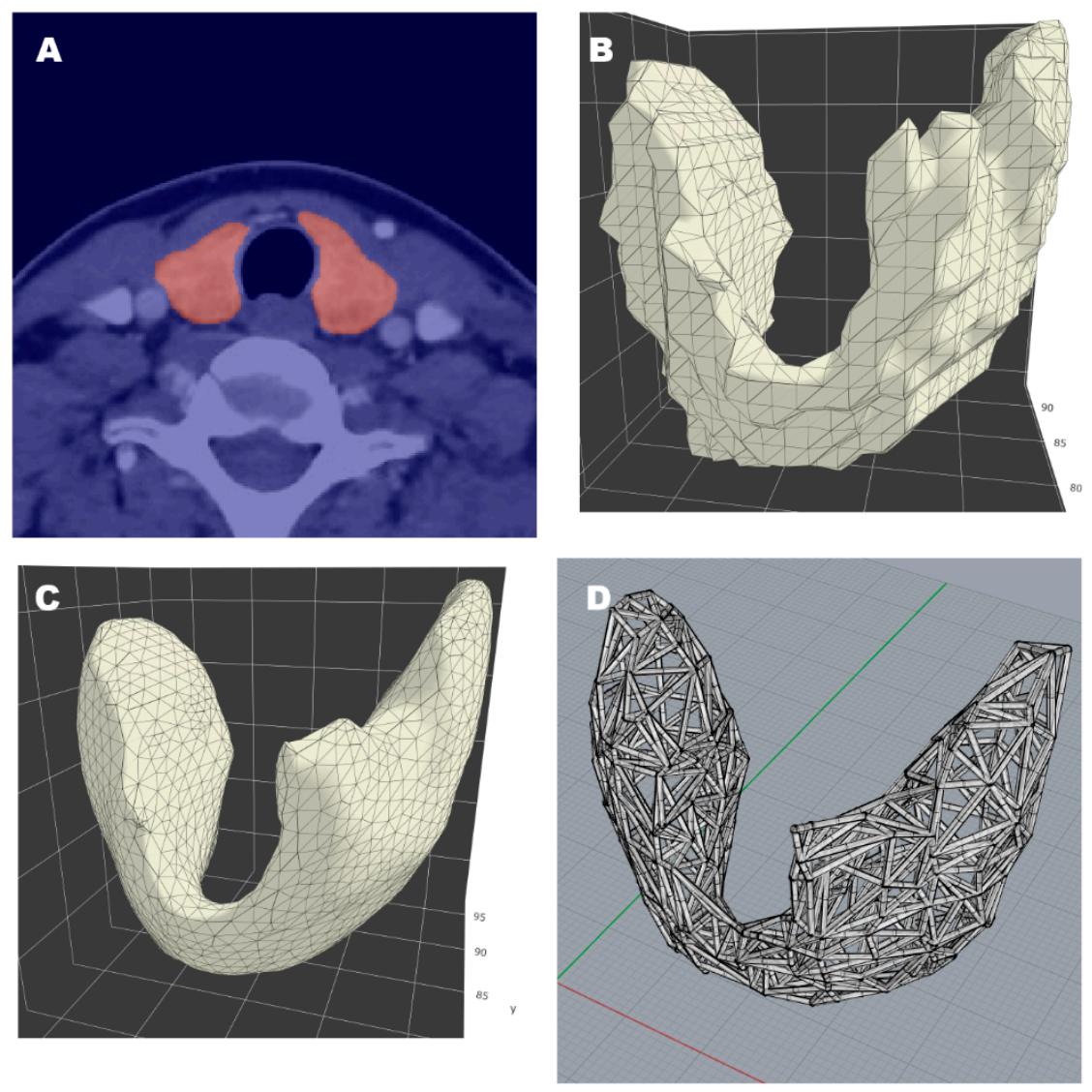

Figure 2. Thyroidal prototype of mesh-work 3D modeling: (A) Segmentation of thyroid gland in tomographic image; (B) reconstruction via triangular mesh; (C) surface smoothing; (D) resultant 3D model.

We engaged a two-color 3D printer (3DWOX 2X; Sindoh, Seoul, Korea) and its slicer software (3DWOX Desktop; Sindoh, Seoul, Korea) for model fabrication. This printer works in fused deposition modeling (FDM) mode, extruding polylactic acid (PLA) as the material of choice. In all processes, the manufacturer's default slicer parameters were unchanged (Table S1). Ultimately, we confirmed the clinical potential of a 3D-printed thyroidal prototype by comparing it with the corresponding thyroidectomy specimen.

\section{Results}

Several organ-tumor models were printed in this manner (Figure 1). By varying mesh densities, disparate structures were distinguishable, as follows: thyroid lobe and trachea (Figure 1C), maxillary bone and teeth (Figure 1D), breast and nipple (Figure 1E), and the three lobes of the right lung (Figure 1F). The mesh style is indicative of tumor occupancy in 3D (even if inside solid organs), without the need of transparent material.

Printed dimensions (relative to actual size), printing time, post-processing time, and cost were evaluated in each organ-tumor model (Table 1). Post-processing time was spent removing model support structures that the slicer program automatically applied. Both printing time and cost significantly correlated with the 3D model size: $1.73 \mathrm{~min} / \mathrm{cm}^{3}$ $(p<0.001)$ and $\$ 0.05 / \mathrm{cm}^{3}(p<0.001)$. 
Table 1. Specifications, production times, and costs of various mesh-type 3D models.

\begin{tabular}{cccccc}
\hline Model & $\begin{array}{c}\text { Dimensions } \\
\mathbf{( c m})\end{array}$ & $\begin{array}{c}\text { Relation } \\
\text { to Actual } \\
\text { Size }\end{array}$ & $\begin{array}{c}\text { Printing } \\
\text { Time }\end{array}$ & $\begin{array}{c}\text { Post- } \\
\text { Processing } \\
\text { Time (min) }\end{array}$ & Cost \\
\hline Thyroid tumor & $5.3 \times 3.0 \times 6.5$ & 1 & $2 \mathrm{~h} 50 \mathrm{~min}$ & 10 & $\$ 2.72$ \\
\hline $\begin{array}{c}\text { Thyroid gland and } \\
\text { adjacent cartilage }\end{array}$ & $5.5 \times 4.3 \times 9.3$ & 1 & $9 \mathrm{~h} 15 \mathrm{~min}$ & 10 & $\$ 8.69$ \\
\hline Maxillary tumor & $7.1 \times 5.7 \times 4.7$ & 1 & $9 \mathrm{~h} 4 \mathrm{~min}$ & 15 & $\$ 8.24$ \\
\hline Parathyroid adenoma & $4.0 \times 3.1 \times 5.5$ & 1 & $5 \mathrm{~h} \mathrm{3} \mathrm{min}$ & 10 & $\$ 3.71$ \\
\hline Breast tumor & $6.2 \times 5.2 \times 5.9$ & $\frac{1}{2}$ & $6 \mathrm{~h} 41 \mathrm{~min}$ & 5 & $\$ 7.78$ \\
\hline Lung nodule & $6.8 \times 8.0 \times 13.5$ & $\frac{1}{2}$ & $23 \mathrm{~h} 4 \mathrm{~min}$ & 40 & $\$ 33.67$ \\
\hline
\end{tabular}

We also compared a 3D-printed model of a thyroid gland with the actual thyroidectomy specimen, shown in Figure 3. There was no observed disparity in glandular size or contour. The model and surgical specimen also corresponded well in terms of tumor location.
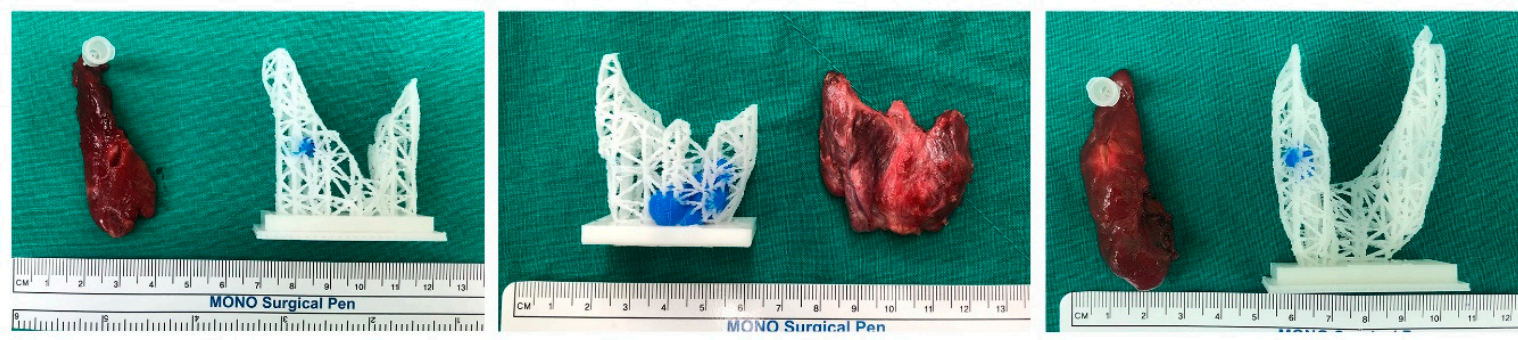

Figure 3. Side-by-side comparison of a 3D-printed thyroidal prototype and a corresponding surgical specimen.

\section{Discussion and Conclusions}

\subsection{Discussion}

In the context of tumors, adequate information and understanding conveyed to a competent patient provides a sound basis for voluntary and rational decision-making [14]. Thus, the primary goal of informed surgical consent is establishing a rudimentary appreciation of key principles, including organ anatomy, physiology, and attributes, tumor location, and potential operative complications [7]. In portraying a solid tumor, capsular breech and invasion of surrounding tissues are other important considerations that a 3D model effectively addresses. A personalized model rendered by a 3D printer reinforces the mutual sense of awareness needed between patient and physician [7]. Only a few studies to date have tested 3D models in patients facing thoracic, kidney, or orthopedic surgeries, but they have regularly shown improved preoperative understanding or satisfaction [7-9]. Still, a number of issues pertaining to cost, resolution, and duration of printing and material texture must be resolved before 3D-printed models become more mainstream [11].

Our novel methodology offers several advantages. The customary segmentation of targeted objects entailed in 3D reconstruction of tomographic images is readily applicable to mesh-type 3D modeling. Hence, newer CT systems are not required and routine CT protocols for specific organs may be utilized. Likewise, an expensive 3D printer is not essential. Even if just two colors are available, tumors and normal structures can be printed in different colors and the density of the same color can be changed to differentiate two or more normal structures. In addition, the solid form can be used together; tumors may be printed as either solid or mesh forms. Solid replicas may provide better visibility of small lesions. Filament requirements (and thus costs) are similarly reduced because the printed organs are largely empty inside. Given a magnitude $\leq 10 \mathrm{~cm}$, most models were fabricated within half a day. Thus, the day after computed tomography or magnetic resonance imaging (MRI) studies were obtained, completed models were feasible. 
There is some concern that mesh-type 3D modeling results do not accurately reflect the organs or the tumors they represent. However, further attempts at refinement may nevertheless impose unwanted steps. For example, CT imaging at 1-mm slice thickness (vs. routine setting) as a means of enhancing replication demands higher radiation exposure. Although not statistically proven, the size and contour of a printed thyroid gland and the tumor situated within it satisfactorily mimicked attributes of the corresponding surgical specimen, confirming this approach as a viable tool for personalized care. However, for applications requiring narrower margins of error (i.e., a surgical guide), the precision of specimen renderings should be studied.

With regard to study limitations, PLA output is clearly of low quality, lacking sophistication [15]. In addition, the printing time of mesh-type 3D models may take longer than the output of the same volume of solid models with the same FDM printer; even half-size printing of a large organ (such as a lung) required nearly an entire day. However, reducing mesh density and stereolithography (STL) file reduction (decimation) could save the printing time. Stringing may also develop as a technical issue, although this is avoidable by controlling printer settings or resorting to non-FDM 3D printing techniques. We could not pursue internally filled models (delineating tumors) to compare costs and printing times, because no transparent or translucent PLA filament was compatible with our printer. Overall, our model depicts actual organ size and tumor location at low levels of resolution. This study remained focused on modeling technique and its clinical import, but a change in parameter default settings might serve to improve the resolution of our models. Optimal settings may even be organ specific. These are lingering issues to be resolved going forward. To truly establish its merit, prospective research is needed.

\subsection{Conclusions}

We have devised a mesh-type 3D modeling technique involving less cost and faster completion than full 3D printing efforts. This approach may facilitate the fabrication of anatomic models for personalized care, helping patients to conceptualize various organs, tumors, and surrounding tissues during informed surgical consent.

Supplementary Materials: The following are available online at https: / / www.mdpi.com/2076-341 7/11/3/1047/s1, Table S1: The manufacturer's default parameters of the slicer software.

Author Contributions: Conceptualization, S.-k.K. and Y.-S.J.; methodology, J.S. and S.-k.K.; software, J.S.; validation, J.S., C.H.R., J.R., and Y.-S.J.; formal analysis, J.S.; data curation, S.Y., C.H.R., J.R.; writing-original draft preparation, J.S., S.Y., C.H.R. and J.R.; writing—review and editing, S.-k.K. and Y.-S.J.; vis-ualization, J.S.; supervision, Y.-S.J.; project administration, Y.-S.J.; funding acquisition, J.S. and Y.-S.J. All authors have read and agreed to the published version of the manuscript.

Funding: This study was supported by a grant from the National Cancer Center in Korea [grant Nos. NCC2031580-1 and NCC2010241-1]. The authors have no financial ties to disclose.

Institutional Review Board Statement: The study was conducted according to the guidelines of the Declaration of Helsinki, and approved by the Institutional Review Board of National Cancer Center, Korea, approved our study protocol (IRB No. NCC2020-0248, date of approval: 2020.09.04).

Informed Consent Statement: Patient consent was waived because no patient data was collected.

Data Availability Statement: The data presented in this study are available on request from the corresponding author. The data are not publicly available because the models were not agreed to be shared publicly.

Conflicts of Interest: JG: CHR, JR, and YSJ are listed on a patent application describing similar methodology and filed by the National Cancer Center, Korea (application no. 10-2020-0000849; filed 03 January 2020). No other authors have conflicts of interest to declare. 


\section{References}

1. Hanson, M.; Pitt, D. Informed consent for surgery: Risk discussion and documentation. Can. J. Surg. 2017, 60, 69-70. [PubMed]

2. Mishra, P.K.; Ozalp, F.; Gardner, R.S.; Arangannal, A.; Murday, A. Informed consent in cardiac surgery: Is it truly informed? J. Cardiovasc. Med. 2006, 7, 675-681. [CrossRef] [PubMed]

3. Villanueva, C.; Talwar, A.; Doyle, M. Improving informed consent in cardiac surgery by enhancing preoperative education. Patient Educ. Couns. 2018, 101, 2047-2053. [CrossRef] [PubMed]

4. Tait, A.R.; Voepel-Lewis, T. Digital multimedia: A new approach for informed consent? JAMA 2015, 313, 463-464. [CrossRef] [PubMed]

5. Rosenfeld, E.H.; Lopez, M.E.; Yangyang, R.Y.; Justus, C.A.; Borges, M.M.; Mathai, R.C.; Karediy, A.; Zhang, W.; Brandt, M.L. Use of standardized visual aids improves informed consent for appendectomy in children: A randomized control trial. Am. J. Surg. 2018, 216, 730-735. [CrossRef] [PubMed]

6. Moseley, T.H.; Wiggins, M.N.; O'sullivan, P. Effects of presentation method on the understanding of informed consent. Br. J. Ophthalmol. 2006, 90, 990-993. [CrossRef] [PubMed]

7. Bernhard, J.C.; Isotani, S.; Matsugasumi, T.; Duddalwar, V.; Hung, A.J.; Suer, E.; Baco, E.; Satkunasivam, R.; Djaladat, H.; Metcalfe, C.; et al. Personalized 3D printed model of kidney and tumor anatomy: A useful tool for patient education. World J. Urol. 2016, 34, 337-345. [CrossRef] [PubMed]

8. Yoon, S.H.; Park, S.; Kang, C.H.; Park, I.K.; Goo, J.M.; Kim, Y.T. Personalized 3D-Printed Model for Informed Consent for Stage I Lung Cancer: A Randomized Pilot Trial. Semin. Thorac. Cardiovasc. Surg. 2019, 31, 316-318. [CrossRef] [PubMed]

9. Zhuang, Y.; Zhou, M.; Liu, S.; Wu, J.; Wang, R.; Chen, C. Effectiveness of personalized 3D printed models for patient education in degenerative lumbar disease. Patient Educ. Couns. 2019, 102, 1875-1881. [CrossRef] [PubMed]

10. Tack, P.; Victor, J.; Gemmel, P.; Annemans, L. 3D-printing techniques in a medical setting: A systematic literature review. Biomed. Eng. Online 2016, 15, 115. [CrossRef] [PubMed]

11. Yan, Q.; Dong, H.; Su, J.; Han, J.; Song, B.; Wei, Q.; Shi, Y. A Review of 3D Printing Technology for Medical Applications. Engineering 2018, 4, 729-742. [CrossRef]

12. Hesamian, M.H.; Jia, W.; He, X.; Kennedy, P. Deep learning techniques for medical image segmentation: Achievements and challenges. J. Digit. Imaging 2019, 32, 582-596. [CrossRef] [PubMed]

13. Dawson-Haggerty, S. Trimesh [Internet]. Available online: https://trimsh.org/ (accessed on 1 March 2020).

14. Lidz, C.W. The therapeutic misconception and our models of competency and informed consent. Behav. Sci. Law 2006, 24, 535-546. [CrossRef] [PubMed]

15. Garcia, J.; Yang, Z.L.; Mongrain, R.; Leask, R.L.; Lachapelle, K. 3D printing materials and their use in medical education: A review of current technology and trends for the future. BMJ Simul. Technol. Enhanc. Learn. 2018, 4, 27-40. [CrossRef] [PubMed] 